\title{
Labelling of rhinitis and hayfever by doctors
}

\author{
Bonnie Sibbald, Elizabeth Rink
}

\begin{abstract}
Factors influencing the labelling of rhinitis by doctors were examined in 7702 adults, aged 16-65 years, registered with a group general practice in south London. Individuals with rhinitis were identified by a postal screening questionnaire. Of the $2969(39 \%)$ subjects who replied, 1309 (44\%) reported rhinitis. A sample of 340 individuals with and 126 without rhinitis was studied, each being interviewed and having allergy skinprick tests. The information given at interview was compared with that recorded in the medical notes for 75 patients with rhinitis, selected at random to include 25 with seasonal symptoms only, 25 with perennial symptoms only, and 25 with both seasonal and perennial symptoms, plus 25 control subjects. Hayfever was defined as seasonal symptoms provoked by grass and a positive skin test response to grass pollen. Subjects with seasonal symptoms were nearly twice as likely as those with perennial symptoms to be labelled as having rhinitis by their doctor. More than a quarter of the subjects fulfilling our definition of hayfever had not been diagnosed as having hayfever. Subjects from the upper social classes were more likely than those from the lower social classes to be labelled as having hayfever, though there were no significant differences between social classes in the prevalence of hayfever according to our definition. It is concluded that rhinitis is frequently underdiagnosed and misdiagnosed. Increased detection of hayfever could account for the steep rise in consultation rates seen in recent decades.
\end{abstract}

The past decade has seen a sharp rise in general practice consultation rates for hayfever, from $11.0 / 1000$ people in 1971 to $19 \cdot 7 / 1000$ in $1981 . .^{12}$ Though rare before the nineteenth century, ${ }^{3}$ hayfever is now among the most common chronic diseases seen in general practice in the United Kingdom, ${ }^{2}$ with an estimated prevalence of $10 \%{ }^{4}$

The possible reasons for this apparent increase in prevalence are not known, but may include a change in consulting behaviour by patients, a change in diagnostic fashion by general practitioners, and a true rise in the prevalence of the disease. There are no reports of longitudinal change in the prevalence of hayfever in the community on the basis of objective diagnostic criteria. The evidence favouring a true increase in prevalence is therefore circumstantial. The consulting behaviour of patients for hayfever has not been studied and conceivably improvements in drug treatment cause more patients to visit the doctor. Fleming and Crombie found an appreciable rise in consultation rates for hayfever between 1970-1 and 1981-2 among the 19 general practices contributing to both National Morbidity Surveys. ${ }^{5}$ They concluded that this reflected an increase in the prevalence of hayfever that could not be explained by changing diagnostic fashion, but as they did not examine doctors' diagnostic preferences the latter cannot be ruled out.

We have examined the labels applied to rhinitis by doctors and the factors associated with their acquisition as part of a community survey of rhinitis among adults in south London.

\section{Methods}

A screening questionnaire designed to identify those with rhinitis was posted during MarchMay 1988 to all patients aged 16-65 years who were registered with a group practice of six in south London. Rhinitis was said to be present when an individual reported a problem with sneezing, rhinorrhoea, or nasal blockage that was not solely associated with upper respiratory infections and had persisted for two or more years. A sample of 640 subjects with rhinitis and 307 subjects without rhinitis was identified from the screening questionnaire and these were invited for more detailed interview during June-August. Rhinitic patients were stratified according to the overall severity and the seasonal-perennial nature of their symptoms to ensure that all types and severities of rhinitis were represented. Those who agreed were interviewed in depth about their symptoms, medical care, and possible risk factors for rhinitis.

Allergy skinprick tests were administered to all consenting rhinitic and non-rhinitic subjects after the interview. Five allergens were used: house dust, house dust mite, mixed grass pollen, mixed tree pollen and cat dander (Bencard), together with glycerol and histamine control solutions. A positive reaction was defined as a weal at least $3 \mathrm{~mm}$ larger than the glycerol control, in the presence of a reaction to the histamine control. Subjects with one or more positive reactions were said to be atopic. Hayfever was said to be present when the skin test response to grass pollen was positive and the subject described 
seasonal symptoms on exposure to grass.

Subjects were asked at interview what label, if any, the doctor had given their condition, whether they had ever consulted the general practitioner specifically about nasal symptoms, and what treatments they had used in the past year. Doctors' labelling was examined in relation to the nature of subjects' symptoms and their skin test sensitivity. Labelling and drug treatment were further examined in relation to whether or not subjects said they had consulted the general practitioner about nasal symptoms.

The possible bias introduced by patients' non-response was investigated by comparing the medical notes of a random sample of 100 subjects who did not participate in the study with those of 100 subjects ( 25 with seasonal symptoms, 25 with perennial symptoms, 25 with both perennial and seasonal symptoms, and 25 without rhinitis) who were interviewed. Data on participants were also used to assess the agreement of information recorded in medical notes with that given at interview.

Data were analysed by means of the Statistical Package for the Social Sciences (SPSS$X)$. The significance of differences between variables, was assessed by the $\chi^{2}$ test. All reported differences are statistically significant at the $5 \%$ level unless otherwise stated.

\section{Results}

The screening questionnaire was mailed to 7702 adults, of whom $2969(39 \%)$ returned a usable questionnaire. Among the non-responders were $113(1 \%)$ with spoiled questionnaires, $1382(18 \%)$ who had moved, and 3238 $(42 \%)$ who failed to respond. Of the 2969 subjects who returned a completed questionnaire, $1309(44 \%)$ fulfilled the criteria for rhinitis. Of the 640 subjects with rhinitis invited for interview, $340(53 \%)$ accepted; of the 307 subjects without rhinitis invited for interview, $126(41 \%)$ accepted. Allergy skinprick tests were carried out in 327 subjects with rhinitis, of whom 208 (64\%) were atopic.

When the medical records of 100 nonresponders were compared with those of 100 subjects interviewed (75 rhinitic and 25 nonrhinitic) no significant differences were found between the groups with regard to sex, marital status, drinking habits, or the proportions with a diagnosis of asthma, eczema, or migraine. Responders, however, were significantly more likely than non-responders to be aged 26 years

Table 2 Influence of social class on the labelling of hayfever ${ }^{\star}$

\begin{tabular}{llll}
\hline & & \multicolumn{2}{l}{ No $(\%)$ of subjects with } \\
\cline { 3 - 4 } Social class & No in class & label of hayfever & hayfever \\
\hline I, II & 160 & $54(34)$ & $56(35)$ \\
III & 118 & $46(39)$ & $39(33)$ \\
IV, V & 35 & $3(9)$ & $7(20)$ \\
Significance of difference among social classes: & $\mathrm{p}<0.01$ & $\mathrm{p}>0 \cdot 20$ \\
\hline
\end{tabular}

*The findings are confined to the 313 rhinitic subjects for whom there were both allergy skinprick test data and social class coding.

tHayfever diagnosed as a positive skin test response to grass pollen and seasonal symptoms provoked by grass.
Table 1 Influence of atopy on the labelling of rhinitis *

\begin{tabular}{lcc}
\hline & \multicolumn{2}{l}{ No $(\%)$ of rhinitic subjects } \\
\cline { 2 - 3 } Doctors' label & Atopic & Non-atopic \\
\hline Hayfever & $99(48)$ & $12(10)^{\star \star \star}$ \\
Rhinitis & $16(8)$ & $11(9)$ \\
Sinusitis & $52(28)$ & $38(32)$ \\
Total & $208(100)$ & $119(100)$ \\
\hline
\end{tabular}

*The table applies to the 327 subjects in whom skin tests were performed. The sums do not total $100 \%$ as not all patients were labelled.

Significance of difference between atopic and non-atopic subjects: ${ }^{\star \star \star} p<0.001$.

or over, a non-smoker, and to come from the upper social classes. They were also more likely to have a diagnosis of rhinitis or sinusitis, which was expected as $75 \%$ of those interviewed had rhinitis.

Subjects with rhinitis were asked at interview about any label given their condition by the doctor. The diagnosis was said to be hayfever in $115(34 \%)$, sinusitis in $97(29 \%)$, and rhinitis in $29(9 \%)$. The labelling of rhinitis and sinusitis showed no significant associations with subjects' social or demographic characteristics, whereas the labelling of hayfever was influenced by atopy and higher social class. Subjects with rhinitis who were atopic were more likely than non-atopic subjects to be labelled as having hayfever (table 1), as were subjects in social classes I, II, and III (table 2). Other social class indicators, notably school leaving age and housing tenure, showed no significant association with the hayfever label.

At interview 106 subjects were found to have hayfever when this was defined as a positive skinprick test response to grass pollen and seasonal symptoms provoked by grass. The condition had been labelled as hayfever by the doctor in $75(71 \%)$. This label had also been given to $31(14 \%)$ of the 221 subjects with rhinitis not fulfilling our criteria for a diagnosis of hayfever. No significant association was found between the prevalence of hayfever (our definition) and social class (table 2), school leaving age, or housing tenure.

Doctors' labelling and drug treatment of rhinitis was examined in relation to whether or not subjects said they had consulted the general practitioner about nasal symptoms. Subjects who had consulted about these were more likely than those who had never done so to be labelled as having hayfever or rhinitis (table 3 ). Nevertheless, among those who had consulted, subjects with seasonal symptoms were twice as likely as those with perennial symptoms to have been labelled by the doctor. Among the subjects who had consulted for their nasal symptoms, those who were labelled as having rhinitis were significantly more likely to be taking antihistamines, topical corticosteroids, or topical cromoglycate for their rhinitis (table 4). Those not having a label of rhinitis were more likely to be taking sympathomimetics or antibiotics for their rhinitis, though these differences did not reach significance.

The agreement between patients and doctors about diagnostic label was investigated by examining the medical records of a random 
Table 3 Labels applied to rhinitic subjects by doctors

\begin{tabular}{lllllll}
\hline & & & \multicolumn{2}{c}{ Doctors' labels (No (\%) of subjects) } \\
\cline { 5 - 7 } Clinical presentation & Patients & $n$ & Hayfever & Rhinitis & Either \\
\hline Seasonal symptoms & All & 195 & $90(46)$ & $17(9)$ & $96(49)$ \\
& Consulters & 129 & $80(62)$ & $16(13)$ & $85(66)$ \\
Perennial symptoms alone & All & 131 & $21(16)$ & $12(9)$ & $31(24)$ \\
& Consulters & 68 & $21(31)$ & $11(16)$ & $30(44)$ \\
Summer seasonal, provoked by grass & All & 119 & $83(70)$ & $11(10)$ & $83(70)$ \\
Summer seasonal, provoked by grass, pollen skin test & Consulters & 86 & $76(88)$ & $11(13)$ & $76(88)$ \\
& All & 104 & $75(72)$ & $10(10)$ & $75(72)$ \\
& Consulters & 75 & $69(92)$ & $10(14)$ & $69(92)$ \\
& & &
\end{tabular}

*The findings are confined to the 326 subjects for whom there was complete information on both symptoms and doctors' labels.

"Consulters" refers to those subjects who had consulted the general practitioner for nasal symptoms.

sample of 75 rhinitic and 25 non-rhinitic subjects. Thirty four $(45 \%)$ of those with rhinitis had a diagnosis of rhinitis or hayfever recorded in their medical records, as did three of the 25 without rhinitis. The agreement between interview data and medical records varied according to the seasonal or perennial nature of the patients' condition ( $p<0.05)$. Of 25 subjects who said that they had only seasonal symptoms at interview, $16(64 \%)$ had a diagnosis of hayfever or rhinitis in their medical records. By comparison, only $17(35 \%)$ of 48 subjects with perennial symptoms had a diagnosis of rhinitis in their medical records.

\section{Discussion}

In this study subjects with perennial symptoms were nearly twice as likely as those with seasonal symptoms to be undiagnosed, and a quarter of the subjects who had hayfever according to our criteria had not been given such a diagnosis. Subjects from the upper social classes were more likely than those from the lower social classes to be labelled as having hayfever, though there were no significant differences between social classes in the prevalence of hayfever diagnosed by our criteria.

We have adopted as our definition of hayfever a positive skinprick test response to grass pollen and summer seasonal symptoms provoked by grass pollen. Subjects who meet these criteria almost certainly have hayfever, but others with the condition may have been excluded because they failed to develop a $3 \mathrm{~mm}$ weal in response to the specific mix of pollens tested or reactivity was localised to the nasal mucosa alone. For this reason and because doctors would not normally carry out skin tests in diagnosing rhinitis, we also examined doctors' labelling in relation to subjects' symptoms

Table 4 Association of doctor's label with drug treatment among patients consulting the general practitioner for nasal symptoms

\begin{tabular}{llc}
\hline & \multicolumn{2}{l}{ Label of hayfever or rhinitis $(N o(\%)$ of subjects) } \\
\cline { 2 - 3 } Current drug treatment & $Y e s(n=119)$ & $N o(n=85)$ \\
\hline Sympathomimetics & $28(24)$ & $30(35)$ \\
Antihistamines & $78(66)$ & $20(24)^{\star \star \star}$ \\
Corticosteroids & $32(27)$ & $7(8)^{\star \star}$ \\
Cromoglycates & $13(11)$ & $1(1)^{\star}$ \\
Antibiotics & $6(5)$ & $10(12)$ \\
Any of the above & $104(88)$ & $65(77)^{\star}$ \\
\hline
\end{tabular}

Significance of difference between labelled and unlabelled patients: ${ }^{\star} p<0.05$; ${ }^{\star \star} p<0.01$ $\star \star \star p<0.001$. alone. It remained true that more than a quarter of the subjects with summer seasonal symptoms provoked by pollen had not been given the diagnosis of hayfever, and that subjects with perennial symptoms were twice as likely as those with seasonal symptoms to be undiagnosed.

The study was conducted in only one group general practice, which means that the diagnostic labels given represent the views of a limited number of doctors. As the migration of patients is high in London, however, labels will have been given by more doctors than those in this one practice. The details of doctors' diagnoses were obtained retrospectively from patients' medical records to avoid the bias of prospective studies, which may heighten doctors' awareness in detection and diagnosis. The response rate was low and the interview sample favoured older, more severely affected patients-a bias that should have made detection and appropriate diagnosis more likely in the participants than in the non-participants. Our findings regarding the underdiagnosis and misdiagnosis of rhinitis may therefore underestimate the true extent of the problem.

The underdiagnosis of rhinitis is explained in part by subjects' failure to consult the general practitioner about their symptoms. Among subjects who did consult, $92 \%$ of those with summer seasonal symptoms provoked by grass pollen were labelled as having hayfever. Even among patients consulting for their nasal symptoms, however, only $44 \%$ of those with perennial symptoms and $66 \%$ of those with seasonal symptoms (any season) were labelled as having rhinitis or hayfever by the doctor. Although doctors may prefer not to label patients whose symptoms are mild, patients regarded their condition as serious enough to bring it to the attention of a doctor.

Labelling has important implications for treatment. Among patients who had consulted the general practitioner for nasal symptoms, those who were labelled as having hayfever or rhinitis were more likely to be taking specific antirhinitic medication such as antihistamines, topical corticosteroids, or topical cromoglycate. Among patients who had not been labelled there was a tendency towards increased use of antibiotics and symptomatic treatment with sympathomimetics.

How social class affected labelling in the present study is not clear because other social indicators, such as school leaving age and 
housing tenure, showed no significant association with the hayfever label. The numbers of subjects in the lower social classes was small, which means that the misclassification of only a small number of cases could explain the observed trend towards underdiagnosis of hayfever in the lower social classes.

The association of hayfever with social class has been noted previously in some studies but not others. In a survey of 12743 British children aged 5 years the prevalence of hayfever declined from $5.3 \%$ in the children of "advantaged" households to $3 \cdot 1 \%$ in the children of "disadvantaged" households. ${ }^{6}$ Similarly, in a community survey of 9226 Americans subjects with allergic rhinitis had a higher annual income than subjects without. ${ }^{7}$ The trend was inconsistent, however, in that subjects in the middle income group had the highest prevalence of allergic rhinitis. There was no significant association between hayfever and socioeconomic factors among 2697 patients attending Danish general practitioners. ${ }^{8}$ In each of these studies the diagnosis of hayfever relied wholly or partly on doctors' labelling of the condition, so the differences could be due to variation in doctors' labelling preferences rather than differences in the prevalence of hayfever.

A similar bias has been noted in doctors' labelling of asthma. Community studies of both adults ${ }^{9}$ and children ${ }^{10}$ suggested that patients with wheezing illness were more likely to be labelled asthmatic if they came from the upper than from the lower social classes.

Underdiagnosis was most apparent for perennial rhinitis but occurred also for seasonal rhinitis. This substantial reservoir of undiagnosed disease means that a shift towards increased detection of hayfever could explain the apparent increase in the prevalence of this condition in past decades. This trend might be further enhanced by the introduction of better drug treatments, which encourage symptomatic patients to attend their general practitioner. Consequently caution is needed in making inferences about the epidemiology of rhinitis based on physicians' diagnoses or data on the use of services.

We would like to thank the Medical Research Council for its generous support of this work; the doctors of the Brocklebank generous support of this work; the doctors of the Brocklebank
Health Centre for giving us access to their patients; and the patients themselves for their kind cooperation. Our thanks go patients themselves for their kind cooperation. Our thanks go
also to Professor H R Anderson for his critical appraisal of all aspects of the work.

1 Royal College of General Practitioners, Offices of Population Censuses and Surveys, and Department of Health and Social Security. Morbidity statistics from general practice 1970-1. Second national study. London: HMSO, 1970-1. Second

2 Royal College of General Practitioners, Offices of Population Censuses and Surveys, and Department of Health and Social Security. Morbidity statistics from general practice 1981-2. Third national study. London: HMSO, 1986:table 18.

3 Emanuel M. Hay fever, a post industrial revolution epidemic: a history of its growth during the 19th century. epidemic: a history of its growth

4 Ewan P. Hay fever. A report of the Royal College of Physicians of London. London: Royal College of Physicians, 1989.

5 Fleming $D$, Crombie D. Prevalence of asthma and hay fever in England and Wales. BMJ 1987;294:279-83.

6 Taylor B, Wadsworth J, Golding J, Butler N. Breast feeding, eczema, asthma, and hayfever. J Epidemiol Community Health 1983;37:95-9.

7 Broder I, Higgins M, Mathews K, Keller J. Epidemiology of asthma and allergic rhinitis in a total community, Tecum seh Michigan. J Allergy Clin Immunol 1974;53:127-38.

8 Weeke ER. Epidemiology of hay fever and perennial allergic rhinitis. Monogr Allergy 1987;21:1-20.

9 Littlejohns P, Ebrahim S, Anderson R. Prevalence and diagnosis of chronic respiratory symptoms in adults. $B M$ 1989;298:1556-60.

10 Peckham C, Butler N. A national study of asthma in childhood. J Epidemiol Community Health 1978;32:79-85. 